\title{
Resonant Coupling of a Bose-Einstein Condensate to a Micromechanical Oscillator
}

\author{
David Hunger, ${ }^{1,2}$ Stephan Camerer, ${ }^{1,2}$ Theodor W. Hänsch, ${ }^{1,2}$ Daniel König, ${ }^{1}$ Jörg P. Kotthaus, ${ }^{1}$ \\ Jakob Reichel, ${ }^{3}$ and Philipp Treutlein ${ }^{1,2, *}$ \\ ${ }^{1}$ Fakultät für Physik, Ludwig-Maximilians-Universität, Schellingstraße 4, 80799 München, Germany \\ ${ }^{2}$ Max-Planck-Institut für Quantenoptik, Hans-Kopfermann-Strasse 1, 85748 Garching, Germany \\ ${ }^{3}$ Laboratoire Kastler Brossel, ENS/UPMC-Paris 6/CNRS, 24 rue Lhomond, F-75005 Paris, France
}

(Received 27 November 2009; published 9 April 2010)

\begin{abstract}
We report experiments in which the vibrations of a micromechanical oscillator are coupled to the motion of Bose-condensed atoms in a trap. The interaction relies on surface forces experienced by the atoms at about $1 \mu \mathrm{m}$ distance from the mechanical structure. We observe resonant coupling to several well-resolved mechanical modes of the condensate. Coupling via surface forces does not require magnets, electrodes, or mirrors on the oscillator and could thus be employed to couple atoms to molecular-scale oscillators such as carbon nanotubes.
\end{abstract}

PACS numbers: $37.10 . \mathrm{Gh}, 03.75 . \mathrm{Kk}, 34.35 .+\mathrm{a}, 85.85 .+\mathrm{j}$

Ultracold atoms can be trapped and coherently manipulated close to a surface using chip-based magnetic microtraps ("atom chips") [1]. This opens the possibility of studying interactions between atoms and on-chip solidstate systems such as micro- and nanostructured mechanical oscillators $[2,3]$. Such oscillators have attracted attention due to the extreme force sensitivity [2] down to the single spin level [4] and the novel manipulation techniques of cavity optomechanics [3]. The question is raised whether the toolbox for quantum manipulation of ultracold atoms could be employed to read out, cool, and coherently manipulate the oscillators' state. Several theoretical proposals have considered the coupling of micro- and nanomechanical oscillators to atoms [5,6], ions $[7,8]$, and molecules [9], and related proposals were made for solidstate two-level systems [10]. They show that sufficiently strong and coherent coupling would enable studies of entanglement, quantum state transfer, and quantum control of mechanical force sensors. In many scenarios involving atomic systems, the coupling relies on local field gradients, calling for very close approach of the atoms to the oscillator. In this respect, ground-state neutral atoms stand out because preparation [11] and coherent manipulation [12] at micrometer distance from a solid surface has already been demonstrated on atom chips. While the intrinsically weak coupling of neutral atoms to the environment enables long coherence times, it makes coupling to solid-state degrees of freedom nontrivial. So far, only first steps have been made to investigate coupling mechanisms experimentally. Recently [13], atoms in a vapor cell were magnetically coupled to a mechanical oscillator. There, thermal motion of the atoms limits the interaction time and the control over the coupling.

In our experiment, we use a Bose-Einstein condensate (BEC) of ${ }^{87} \mathrm{Rb}$ atoms [14] as a sensitive local probe for oscillations of a micromechanical cantilever. Benefiting from its small spatial extent $(<300 \mathrm{~nm})$ and high positioning reproducibility $(<6 \mathrm{~nm})$ in a magnetic microtrap, we place the BEC at about $1 \mu \mathrm{m}$ distance from the surface of the cantilever. At such small distance, the magnetic trapping potential $U_{m}$ is substantially modified by the surface potential $U_{s}=U_{\mathrm{CP}}+U_{\mathrm{ad}}$. It consists of the CasimirPolder $(\mathrm{CP})$ potential $U_{\mathrm{CP}}[11,15,16]$ and an additional potential $U_{\mathrm{ad}}$ due to surface inhomogeneities or contamination [17]. In the direction perpendicular to the surface, the combined potential is [see Fig. 1(c)]

$$
\begin{aligned}
U[z] & =U_{m}+U_{\mathrm{CP}}+U_{\mathrm{ad}} \\
& \approx \frac{1}{2} m \omega_{z, 0}^{2}\left(z-z_{t, 0}\right)^{2}-\frac{C_{4}}{\left(z-z_{c}\right)^{4}}+U_{\mathrm{ad}}\left[z-z_{c}\right] .
\end{aligned}
$$

Here, $z_{c}$ is the position of the cantilever surface, $C_{4}$ the $\mathrm{CP}$ coefficient, and $m$ the atomic mass. Like $U_{\mathrm{CP}}, U_{\mathrm{ad}}$ is attractive and quickly decays with atom-surface distance. The main effect of $U_{s}$ is to reduce the potential depth $U_{0}$ [see Fig. 1(c)] [11]. Additionally, it shifts the trap frequency from $\omega_{z, 0}$ in the unperturbed magnetic trap to $\omega_{z} \approx$ $\left(\omega_{z, 0}^{2}+\frac{1}{m} \frac{\partial^{2} U_{s}}{\partial z^{2}}\right)^{1 / 2}$ and the trap center from $z_{t, 0}$ to $z_{t} \approx$ $z_{t, 0}-\frac{1}{m \omega_{z}^{2}} \frac{\partial U_{s}}{\partial z}$ [18]. When the cantilever oscillates, $U_{s}$ becomes time-dependent and leads to a modulation of $U_{0}, z_{t}$, and $\omega_{z}$ at the cantilever frequency $\omega_{m}$. We show that this excites atomic motion, which can be detected most simply via trap loss across the barrier $U_{0}$. The coupling depends strongly on the trap parameters and shows resonant behavior if $\omega_{m}$ matches the frequency of a collective mechanical mode of the BEC. This can be used to control the interaction efficiently.

Our setup integrates a SiN micro-cantilever of dimensions $(l, w, t)=(200,40,0.45) \mu \mathrm{m}, \mathrm{Au} / \mathrm{Cr}$ metallized upper side, and fundamental resonance frequency $\omega_{m} / 2 \pi=$ $10 \mathrm{kHz}$ on an atom chip [see Fig. 1(a) and 1(b) and [19]). Atoms can be trapped and positioned near the cantilever in a cigar-shaped, dimple-type Ioffe trap [14]. We prepare BECs of typically $N=2 \times 10^{3}$ atoms in state $\left|F=2, m_{F}=2\right\rangle$ without discernible thermal component 
a)

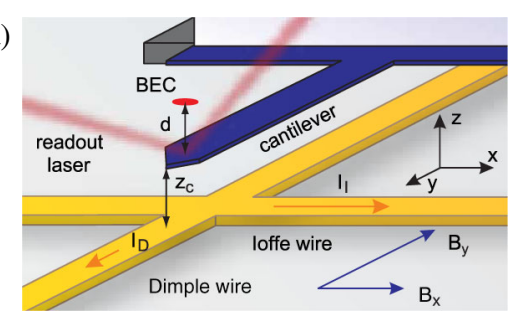

b)

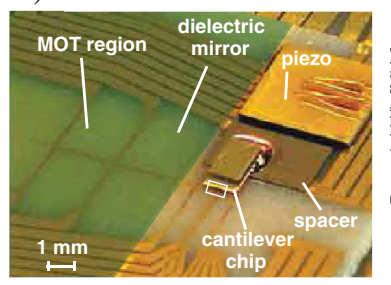

c)

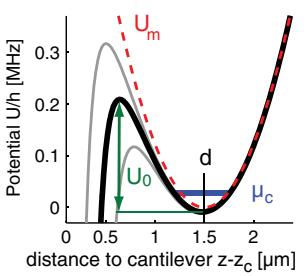

FIG. 1 (color online). (a) Micro-cantilever on a chip with wires for magnetic trapping of atoms. Cantilever vibrations can be independently probed with a readout laser [19]. (b) Photograph of the atom chip showing the magneto-optical trap (MOT) loading region and the cantilever subassembly with a piezo for cantilever excitation. Rectangle: region shown in (a). (c) Potential $U=U_{m}+U_{s}$ for trap parameters as in Fig. 3(a). Dashed red line: magnetic potential $U_{m}$. The surface potential $U_{s}$ reduces the trap depth to $U_{0}$. Cantilever oscillations modulate the potential, thereby coupling to atomic motion. Gray lines: $U$ during the extremum positions of the cantilever for an oscillation amplitude $a=120 \mathrm{~nm}$. Blue line: BEC chemical potential $\mu_{c}$ for 600 atoms.

in a trap at a distance $d=16.6 \mu \mathrm{m}$ from the cantilever. At this distance we observe no influence of the surface.

As a prerequisite for dynamical coupling, we use a method similar to Ref. [11] to determine the range of atom-cantilever distances $d=z_{t, 0}-z_{c}$ where the atoms are affected by $U_{s}$. In these measurements, the cantilever is undriven. We compress the trapping potential to $\omega_{z} / 2 \pi=$ $10 \mathrm{kHz}(5 \mathrm{kHz})$, resulting in a BEC radius of $290 \mathrm{~nm}$ $(430 \mathrm{~nm})$, and ramp adiabatically within $1 \mathrm{~ms}(3 \mathrm{~ms})$ to a set value of $d$ close to the cantilever surface. The atoms are held there for an interaction time $t_{h}=1 \mathrm{~ms}$ during which some of the atoms are lost because of the reduced $U_{0}$. The atoms are subsequently ramped back into a relaxed trap at large distance, where the remaining atom number $N_{r}$ is determined by absorption imaging [14]. Figure 2 shows the remaining fraction $\chi=N_{r} / N$ as a function of $d$. The data shows that we can reproducibly prepare atoms at submicrometer distance from the cantilever. We estimate the positioning reproducibility by measuring the atom number noise on the slope of a surface loss curve at $d=1.3 \mu \mathrm{m}$. A worst case estimate that attributes all the noise to fluctuations of $z_{t, 0}$ yields $\Delta z_{t, 0}=6 \mathrm{~nm}$ rms.

Taking advantage of the suspended structure, we can perform surface loss measurements on both sides of the cantilever, using the atoms as a "caliper" that measures the effective cantilever thickness including $U_{s}$. Comparing the data with a simulation of $U$ allows us to calibrate $d$ to

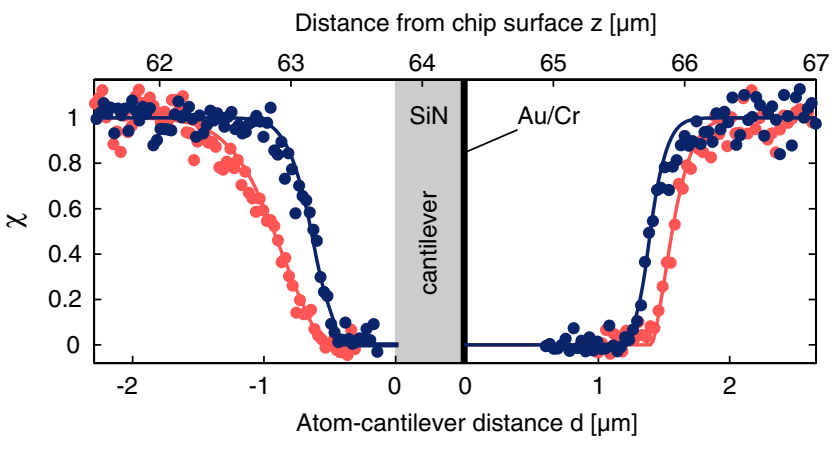

FIG. 2 (color online). Fraction $\chi$ of atoms remaining in the trap after $t_{h}=1 \mathrm{~ms}$ at distance $d$ from the cantilever surface. Blue (red) data points correspond to a trap with $\omega_{z} / 2 \pi=$ $10.0 \mathrm{kHz}(5.1 \mathrm{kHz})$. Solid lines: fit with a simple model $[11,19]$. The extracted cantilever position is shown.

$\pm 160 \mathrm{~nm}$ and to obtain information about $U_{s}$, because $U_{m}$ is very well known. In our analysis, we exploit that $\chi=0$ corresponds to the values of $d$ where the trap has vanished $\left(U_{0}=0\right)$ in good approximation. Alternatively, we employ a model for the surface loss similar to [11] to describe the observed $\chi(d)$, yielding comparable results [19]. The data cannot be explained by $U_{\mathrm{CP}}$ alone but requires $U_{\mathrm{ad}} \gg U_{\mathrm{CP}}$ on at least one side of the cantilever. From measurements of dynamical atom-cantilever coupling (see below) we find that $U_{s}$ is significantly stronger on the metallized side. The data is consistently explained by potentials $U_{\mathrm{ad}}=$ $-C_{\text {ad }} /\left(z-z_{c}\right)^{4}$ with $C_{\text {ad }}=(2 \pm 1) \times 10^{2} C_{4}$ on the metallized side and $C_{\mathrm{ad}}=(10 \pm 10) C_{4, d}$ on the dielectric side. We use the coefficient $C_{4}=3 \hbar c \alpha /\left(32 \pi^{2} \epsilon_{0}\right)$ of a perfect conductor, with $\alpha=5.26 \times 10^{-39} \mathrm{Fm}^{2}$ the ${ }^{87} \mathrm{Rb}$ groundstate polarizability. On the dielectric side, $C_{4, d}=$ $C_{4} \frac{\epsilon-1}{\epsilon+1} \Phi(\epsilon)$, with $\epsilon=4.0$ and $\Phi(\epsilon)=0.77$ for SiN [20]. A likely origin of $U_{\text {ad }}$ is the stray field of surface adsorbates $[17,19]$.

We now describe our main experiments, where cantilever oscillations are coupled to the motion of the atoms nearby. We excite the cantilever with the piezo at frequency $\omega_{p}$. When $\omega_{p}$ is resonant with the cantilever's fundamental out-of-plane mode at $\omega_{m}=2 \pi \times 10 \mathrm{kHz}$, the cantilever oscillates with an amplitude $a$ of typically several tens of $\mathrm{nm}$. We prepare BECs on the metallized side at $d=1.5 \mu \mathrm{m}$ in a trap with $\omega_{z} / 2 \pi=10.5 \mathrm{kHz}$, so that $\omega_{z} \approx \omega_{m}$, and let them interact with the vibrating cantilever. When $\omega_{p}$ is scanned from shot to shot of the experiment, a sharp resonance in the remaining atom number is observed for $\omega_{p}=\omega_{m}$; see Fig. 3(a). This shows that we can use the atoms for cantilever readout. Note that $a$ is more than 1 order of magnitude smaller than $d$, and the cantilever does not touch the atomic cloud. The surface potential of the oscillating cantilever modulates $z_{t}$ with an amplitude $\delta z_{t}=10 \mathrm{~nm}(4 \mathrm{~nm})$ for $a=120 \mathrm{~nm}(50 \mathrm{~nm})$, thereby exciting coherent motion of the atomic center of mass (c.m.). For large c.m. amplitudes the anharmonicity 

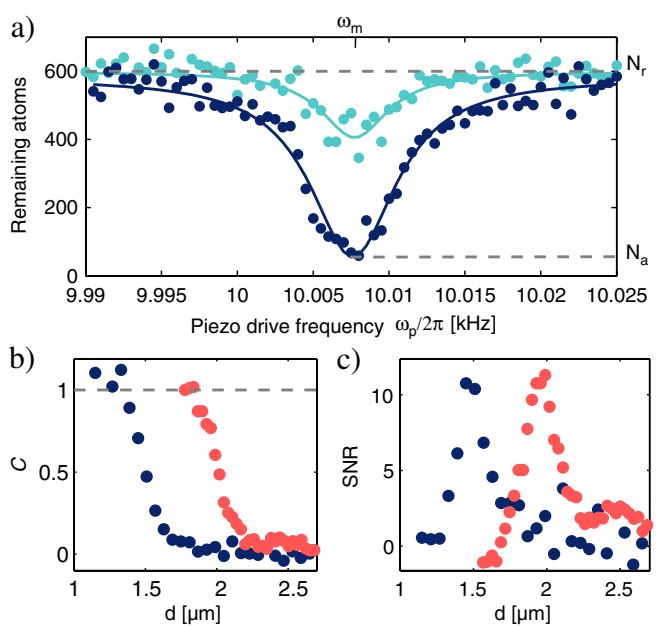

FIG. 3 (color online). (a) Remaining atoms after $t_{h}=3 \mathrm{~ms}$ in a trap with $\omega_{z} / 2 \pi=10.5 \mathrm{kHz}$ at $d=1.5 \mu \mathrm{m}$ from the driven cantilever, for varying drive frequency $\omega_{p}$. The dark (light) blue circles correspond to a cantilever amplitude $a=120 \mathrm{~nm}$ $(50 \mathrm{~nm})$ on resonance. Solid lines: Lorentzian fits with $6 \mathrm{~Hz}$ FWHM, corresponding to the width of the cantilever resonance. (b) and (c) Contrast $C$ and signal to noise ratio SNR of the observed atomic signal as a function of $d$, for constant $a=$ $90 \mathrm{~nm}$ and $\omega_{p}=\omega_{m}$. Blue (red) data points correspond to $\omega_{z} / 2 \pi=10.5 \mathrm{kHz}(5.0 \mathrm{kHz})$ and $t_{h}=3 \mathrm{~ms}(20 \mathrm{~ms})$.

of the deformed trap and the reduced $U_{0}$ convert this motion into heating and loss.

Figure 3(b) shows the dependence of the atomic signal on $d$ for constant $a=90 \mathrm{~nm}$ and $\omega_{p}=\omega_{m}$. We show the contrast $C=\left(N_{r}-N_{a}\right) / N_{r}$, where $N_{a}\left(N_{r}\right)$ is the remaining atom number with (without) resonant piezo excitation of the cantilever. We determine the signal visibility by the signal to noise ratio $\mathrm{SNR}=\left(N_{r}-N_{a}\right) / \sigma$, with $\sigma=32$ the overall noise observed without cantilever driving; see Fig. 3(c). The strong variation of the signal over a few hundred nm matches with the range of $d$ where $U_{s}$ modifies the trapping potential noticeably. We perform similar measurements in a trap resonant with a mode at $\omega_{m}=2 \omega_{z}$ (see below) for longer $t_{h}$ and find comparable behavior at larger distance. If we choose $d$ such that SNR is maximized, we observe a nearly linear dependence $C \propto a$ for $C<1$ and find $\delta z_{t} \propto a$ in the corresponding simulation. We observe the coupling on both sides of the cantilever. Comparison of measurements at similar $U_{0}$ shows that $C / a$ is a factor of $3.2 \pm 0.6$ larger on the metallized side. Because $C / a \propto$ $\partial^{2} U_{s} / \partial^{2} z$ this can be explained by a stronger $U_{s}$ on this side, and combined with loss measurements as in Fig. 2 we can quantitatively infer the strength of $U_{s}$ on both sides [19].

The BEC can be regarded as a mechanical oscillator prepared in the quantum-mechanical ground state. Because of atomic collisions, it has a nontrivial spectrum of collective mechanical modes [21,22]. To demonstrate that the cantilever can be coupled selectively to different BEC modes, we measure the dependence of the atomic response on $\omega_{z}$. The cantilever is excited to constant amplitude and coupled to the BEC on the metallized side. In Fig. 4 we show how the observed atomic SNR changes when we scan $\omega_{z}$. The measured spectrum shows strong resonances at $\omega_{m}=\omega_{z}$ and $\omega_{m}=2 \omega_{z}$. They correspond, respectively, to the atomic c.m. mode and the high frequency $m_{l}=0$ collective mode of the BEC in our cigar-shaped trap $[21,22]$. In our trap, the latter coincides with the breathing mode of the thermal component of the gas. The mode at $\omega_{z}$ $\left(2 \omega_{z}\right)$ is excited by the cantilever through modulation of $z_{t}$ $\left(\omega_{z}\right)$ and we calculate a modulation amplitude of $\delta z_{t}=$ $7 \mathrm{~nm}\left(\delta \omega_{z}=2 \pi \times 150 \mathrm{~Hz}\right)$. For the resonance at $\omega_{m}=$ $2 \omega_{z}$, we observe a linewidth of only $60 \mathrm{~Hz}$, corresponding to a quality factor of $\approx 100$. Because of the trap anharmonicity, a thermal component can lead to a broadening of the resonances. This could explain the line shape of the c.m. mode, where we expect a larger thermal component due to stronger heating at the higher trap frequency [19]. Next to the resonances, we observe reproducible "antiresonances" where the atomic response is suppressed by a factor of 20 . This can be used to switch the coupling on and off. Yet, at this point, we have no clear explanation for their origin. Furthermore, we find up to four weaker resonances at frequencies $\omega_{m}=(1.6,1.8,2.1,2.4) \omega_{z}$. The first resonance can be identified with the $\left|m_{l}\right|=2$ quadrupole mode of the BEC [21], whose frequency is given by $\omega_{m}=$ $\omega_{z} \sqrt{2\left(1+E_{\mathrm{kin}, \perp} / E_{\mathrm{pot}, \perp}\right)}$ [22]. We calculate the BEC kinetic energy $E_{\mathrm{kin}, \perp}$ and potential energy $E_{\mathrm{pot}, \perp}$ in the radial direction as in [23] for 1100 atoms, which reproduces the measured mode frequency. At smaller $d$, we observe broadening of the resonances, and the resonance at $\omega_{m}=$ $1.6 \omega_{z}$ becomes stronger than that at $\omega_{m}=2 \omega_{z}$.

We have used trap loss as the simplest way to detect BEC dynamics induced by the coupling. Measurements as

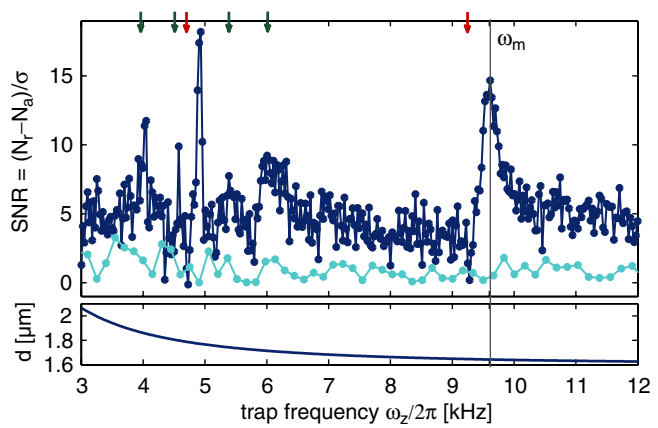

FIG. 4 (color online). Top graph: BEC response as a function of $\omega_{z}$ for constant $a=180 \mathrm{~nm}$ and $t_{h}=20 \mathrm{~ms}$ (dark blue). Data points are connected to guide the eye. Light blue: reference measurement without piezo excitation. We observe two major resonances at $\omega_{m}=\omega_{z}$ and $\omega_{m}=2 \omega_{z}$, up to four smaller ones (green arrows), and reproducible antiresonances (red arrows). Because of cantilever aging, $\omega_{m} / 2 \pi=9.68 \mathrm{kHz}$ in this measurement. Bottom graph: Set values of $d$, chosen such that $N_{r}\left(\omega_{z}\right) \approx$ const $\left(N_{r}(10 \mathrm{kHz})=700, N_{r}(5 \mathrm{kHz})=1100\right)$ and $N_{a}$ does not saturate. 
in Fig. 3(a) yield a minimum resolvable rms cantilever amplitude of $a_{\mathrm{rms}}=13 \pm 4 \mathrm{~nm}$ for $\mathrm{SNR}=1$ without averaging. This value is limited by the strong anharmonicity of the trap, and by the short trap lifetime of $18 \mathrm{~ms}$ (55 ms) for $\omega_{z}=2 \pi \times 10 \mathrm{kHz}(5 \mathrm{kHz})$ due to three-body collisional loss and technical heating. Anharmonicity gives rise to dephasing and thereby limits the cloud amplitude for a given cantilever amplitude. For trap loss to occur, the cantilever has to drive the BEC to large-amplitude oscillations with $\sim 10^{3}$ phonons. By contrast, BEC amplitudes down to the single phonon level could be observed by direct imaging of the motion. A coherent state $|\alpha\rangle$ of the c.m. mode of $N=100$ atoms with $\alpha=1$ released from a relaxed detection trap with $\omega_{z}=2 \pi \times 100 \mathrm{~Hz}$ has an amplitude of $\sqrt{2 \hbar \omega_{z} / m N} \alpha t=400 \mathrm{~nm}$ after $t=4 \mathrm{~ms}$ time of flight. This is about $10 \%$ of the BEC radius and could be resolved by absorption imaging with improved spatial resolution. Assuming that the coupling is linear in $a_{\mathrm{rms}}$, we estimate that $a_{\mathrm{rms}}=0.2 \mathrm{~nm}$ would excite the BEC to $\alpha=1$ within $t_{h}=20 \mathrm{~ms}$ and could thus be detected. This would allow to observe the thermal motion of our cantilever, which has a relatively large effective mass $M=5 \mathrm{ng}$ and correspondingly small rms thermal amplitude $a_{\mathrm{th}}=\sqrt{k_{B} T / M \omega_{m}^{2}}=0.4 \mathrm{~nm}$, where $T=300 \mathrm{~K}$ is the cantilever temperature.

Our experiment is a first demonstration of mechanical coupling between a resonator and ultracold atoms. The coupling relies on fundamental atom-solid-state interactions and does not require fabrication of magnets, electrodes, or mirrors on the oscillator. It could thus serve as the connecting element between atoms and molecularscale oscillators such as carbon nanotubes [24,25]. A single-wall nanotube of $15 \mu \mathrm{m}$ length has $\omega_{m} / 2 \pi=$ $20 \mathrm{kHz}$ and $M=2 \times 10^{-17} \mathrm{~g}$, resulting in $a_{\mathrm{th}}=4 \mu \mathrm{m}$ at $T=300 \mathrm{~K}$ and a quantum-mechanical ground-state amplitude of $a_{\mathrm{qm}}=\sqrt{\hbar / 2 M \omega_{m}}=0.2 \mathrm{~nm}$. This could potentially be detected with the BEC. The surface potential of the nanotube is expected to be a factor $\sim 20$ weaker than $U_{\mathrm{CP}}$ of a bulk conductor [26], which could be compensated to some extent by closer approach of the atoms to the nanotube. Alternatively, electrostatic charging of the nanotube could increase the coupling. It is interesting to study whether such a coupled atom-nanotube system could approach the strong coupling regime [8]. The degree of control over atoms close to a surface demonstrated here is an important ingredient also for coupling schemes that rely on functionalized cantilevers [6]. Furthermore, extending the method of using atoms as a caliper, ultracold atoms can serve as a three-dimensional scanning probe [27], permitting to map out weak electromagnetic fields and surface potentials even inside holes and excavations. This could provide new insights into static and dynamic properties of micro and nano objects.

We acknowledge helpful discussions with T. Steinmetz, I. Favero, P. Böhi, and M. Riedel. This work was supported by the Nanosystems Initiative Munich. T. W. H. gratefully acknowledges support by the Max Planck Foundation. D. K. and J.P.K. acknowledge financial support from QNEMS.

*To whom correspondence should be addressed. Present address: Department of Physics, University of Basel, CH-4056 Basel, Switzerland. philipp.treutlein@unibas.ch

[1] J. Fortágh and C. Zimmermann, Rev. Mod. Phys. 79, 235 (2007).

[2] K. C. Schwab and M. L. Roukes, Phys. Today 58, No. 7, 36 (2005), and references therein.

[3] T. J. Kippenberg and K. Vahala, Science 321, 1172 (2008), and references therein.

[4] D. Rugar, R. Budakian, H. J. Mamin, and B. W. Chui, Nature (London) 430, 329 (2004).

[5] D. Meiser and P. Meystre, Phys. Rev. A 73, 033417 (2006); C. Genes, D. Vitali, and P. Tombesi, Phys. Rev. A 77, 050307(R) (2008); H. Ian et al., Phys. Rev. A 78, 013824 (2008); K. Hammerer et al., Phys. Rev. Lett. 102, 020501 (2009).

[6] P. Treutlein et al., Phys. Rev. Lett. 99, 140403 (2007).

[7] D. J. Wineland et al., J. Res. Natl. Inst. Stand. Technol. 103, 259 (1998); W. K. Hensinger et al., Phys. Rev. A 72, 041405(R) (2005).

[8] L. Tian and P. Zoller, Phys. Rev. Lett. 93, 266403 (2004).

[9] S. Singh et al., Phys. Rev. Lett. 101, 263603 (2008).

[10] A. D. Armour, M. P. Blencowe, and K. C. Schwab, Phys. Rev. Lett. 88, 148301 (2002); P. Rabl et al., Phys. Rev. B 79, 041302(R) (2009).

[11] Y. Lin et al., Phys. Rev. Lett. 92, 050404 (2004).

[12] P. Treutlein et al., Phys. Rev. Lett. 92, 203005 (2004).

[13] Y.-J. Wang et al., Phys. Rev. Lett. 97, 227602 (2006).

[14] W. Hänsel, P. Hommelhoff, T. W. Hänsch, and J. Reichel, Nature (London) 413, 498 (2001).

[15] D. M. Harber et al., Phys. Rev. A 72, 033610 (2005).

[16] H. Bender et al., arXiv:0910.3837.

[17] J. M. Obrecht, R. J. Wild, and E. A. Cornell, Phys. Rev. A 75, 062903 (2007)

[18] M. Antezza, L. P. Pitaevskii, and S. Stringari, Phys. Rev. A 70, 053619 (2004).

[19] See supplementary material at http://link.aps.org/ supplemental/10.1103/PhysRevLett.104.143002 for further details.

[20] Z.-C. Yan, A. Dalgarno, and J. F. Babb, Phys. Rev. A 55, 2882 (1997).

[21] S. Stringari, Phys. Rev. Lett. 77, 2360 (1996).

[22] T. Kimura, H. Saito, and M. Ueda, J. Phys. Soc. Jpn. 68, 1477 (1999).

[23] A. Muñoz Mateo and V. Delgado, Phys. Rev. A 75, 063610 (2007).

[24] P. Poncharal et al., Science 283, 1513 (1999).

[25] B. Babić et al., Nano Lett. 3, 1577 (2003).

[26] R. Fermani, S. Scheel, and P. L. Knight, Phys. Rev. A 75, 062905 (2007).

[27] S. Aigner et al., Science 319, 1226 (2008). 